\title{
Le défi de la prise en charge des personnes issues de la migration
}

Kevin Morisoda, Vishnuga Kanagaratnam ${ }^{b}$, Flora Gobet ${ }^{b}$, Julia Baldwin ${ }^{b}$, Joud Al-Kaarb, Léa Guarinob, Ariane Paulib, Patrick Bodenmannc

a Centre universitaire de médecine générale et santé publique, Unisanté, département Vulnérabilités et médecine sociale, assistant de recherche et doctorant à I'Université de Lausanne; ${ }^{b}$ Université de Lausanne, faculté de biologie et médecine, étudiantes en Master de médecine; ${ }^{c}$ Centre universitaire de médecine générale et santé publique, Unisanté, département Vulnérabilités et médecine sociale, médecin chef et Professeur associé à l'Université de Lausanne

En Suisse, tout le monde n'est pas égal face au système de santé. Les personnes issues de la migration sont particulièrement concernées: le contexte socio-culturel, la barrière linguistique et financière, le manque d'information et parfois la méfiance constituent pour elles des freins à l'accès aux soins. Sur mandat de la Confédération, Unisanté a organisé un Symposium romand sur l'équité des soins, avec l'asile en focus. Le but est de mieux intégrer les migrants dans notre système de santé et de davantage thématiser cette problématique au niveau national.

L'équité en santé, longtemps parent pauvre des systèmes de santé et de la recherche, est décrite par l'Organisation mondiale de la Santé (OMS) comme «l'absence de différences évitables ou remédiables entre différents groupes de personnes, qu'ils soient définis selon des critères sociaux, économiques, démographiques ou géographiques» [1]. Elle commence cependant à prendre de plus en plus d'importance lors de décisions concernant les politiques de santé [2-4]. En effet, l'efficience et la qualité ne suffisent pas à évaluer correctement les performances d'un système de santé et seule une approche intégrative de l'équité en santé permettra de limiter les iniquités entre différentes populations ou minorités [5]. En Suisse également, les préoccupations concernant l'équité en santé se font de plus en plus importantes et les données manquent. L’Office fédéral de la santé

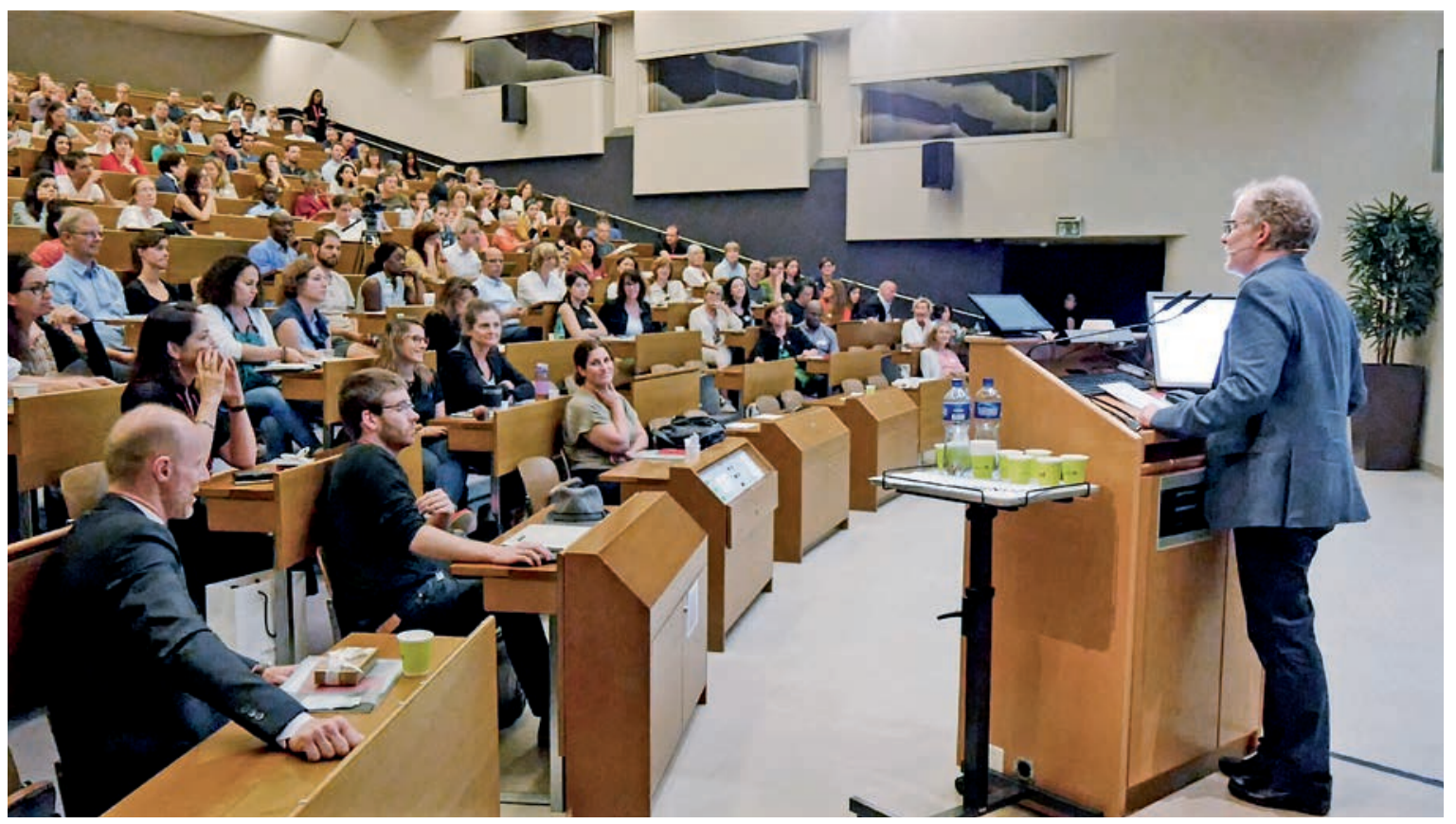

Assemblée nombreuse lors du Symposium, avec le Prof. Patrick Bodenmann à la tribune. (@) Albinfo.ch) 
publique (OFSP) a ainsi décidé de mandater le centre universitaire de médecine générale et santé publique (Unisanté) afin de renforcer l'enseignement sur cette thématique au niveau romand. Grâce à son Département Vulnérabilités et médecine sociale, Unisanté joue un rôle clé d'expertise dans le domaine de l'équité des soins au niveau national [6].

\section{Première conférence du genre}

C'est dans ce contexte qu'a eu lieu en juin le premier Symposium romand sur l'équité des soins, avec pour thème «l'asile aujourd'hui», mandaté et financé par l'OFSP. Près de 250 personnes y ont participé. Une première partie, sous forme de conférence, visait à dresser un panorama descriptif et théorique des questions d'équité en lien avec la migration et l'asile. Animés par des acteurs clés de la prise en charge des migrants, différents ateliers ont été organisés afin de discuter des enjeux concernant l'équité des soins liés à une sous-population de migrants (femmes, enfants), une situation particulière (prisons, urgences, hébergement) ou un enjeu clinique important (santé mentale).
Lors de ces ateliers, trois questions ont été posées aux intervenants afin de cibler les discussions et faire ressortir les éléments essentiels de l'équité en santé en lien avec la migration:

- Quels sont les enjeux en matière d'équité en santé?

- Quelles sont les barrières principales de l'équité en santé?

- Quelles sont les solutions permettant d'améliorer l'équité en santé?

Pour chacune de ces questions, les participants étaient invités à réfléchir sur trois échelles: l'échelle de l'individu, de l'institution et de la société.

\section{Définir les enjeux, solutions et barrières}

La deuxième partie du Symposium a servi à synthétiser le contenu de ces ateliers afin de ressortir la quintessence des propos recueillis tout au long des discussions. Le principal constat est le suivant: malgré des thématiques variées, de nombreux points communs ont émergé des ateliers, mettant ainsi en lumière les enjeux, solutions et barrières principaux de l'équité des soins chez les personnes issues de la migration: la

\begin{tabular}{|c|c|c|c|}
\hline & Individu & Institution & Société \\
\hline Enjeux & $\begin{array}{l}\text { - Contexte social } \\
\text { (isolement, clandestinité) }\end{array}$ & $\begin{array}{l}\text { - Education } \\
\text { - Formation du personnel }\end{array}$ & $\begin{array}{l}\text { - Cadre légal } \\
\text { - Contexte social }\end{array}$ \\
\hline $\begin{array}{l}\text { Quels sont les enjeux } \\
\text { en matière d'équité en } \\
\text { santé dans le contexte } \\
\text { de la migration et de } \\
\text { l'asile? }\end{array}$ & $\begin{array}{l}\text { - Langue } \\
\text { - Culture } \\
\text { - Littératie en santé } \\
\text { - Vulnérabilité liées aux violences } \\
\text { subies en amont, pendant et après } \\
\text { le trajet migratoire } \\
\text { - Accessibilité aux soins } \\
\text { - Méfiance du système } \\
\text { - Adhésion thérapeutique et suivi }\end{array}$ & $\begin{array}{l}\text { - Compétences transculturelles } \\
\text { - Données de la littérature } \\
\text { - Ressources financières (tarification } \\
\text { médicale, financement de l'inter- } \\
\text { prétariat) }\end{array}$ & $\begin{array}{l}\text { - Enjeu financier: financement des soins, } \\
\text { - Cononcement aux soins, franchises } \\
\text { - Volonté politique } \\
\text { - Média et opinion public } \\
\text { - Santé publique } \\
\text { - Culture }\end{array}$ \\
\hline $\begin{array}{l}\text { Barrières } \\
\text { Quelles sont les bar- } \\
\text { rières principales de } \\
\text { l'équité en santé dans } \\
\text { le contexte de la } \\
\text { migration et de l'asile? }\end{array}$ & $\begin{array}{l}\text { - Barrière financière (financement } \\
\text { des soins et de l'interprétariat) } \\
\text { - Barrière linguistique } \\
\text { - Découragement et fatalisme } \\
\text { - Stéréotypes } \\
\text { - Inaccessibilité des médecins } \\
\text { - Mauvaise adhérence aux traitements } \\
\text { et suivi }\end{array}$ & $\begin{array}{l}\text { - Manque de ressources financières } \\
\text { - Administration: règles institution- } \\
\text { nelles, démarches administratives, } \\
\text { structures inadaptées } \\
\text { - Iniquités des options thérapeutiques } \\
\text { proposées } \\
\text { - Inaccessibilité de l'interprétariat } \\
\text { - Discontinuité des soins } \\
\text { - Préjugés, biais de perception et } \\
\text { stéréotypes }\end{array}$ & $\begin{array}{l}\text { - Parlement fédéral actuel } \\
\text { - Recommandations suisses inadaptées } \\
\text { - Manque de financement } \\
\text { - Méconnaissance des enjeux et amal- } \\
\text { games } \\
\text { - Inaccessibilité géographique à certaines } \\
\text { structures de soins } \\
\text { - Stéréotypes, stigmatisation }\end{array}$ \\
\hline $\begin{array}{l}\text { Solutions } \\
\text { Quelles sont les solu- } \\
\text { tions permettant } \\
\text { d'améliorer l'équité en } \\
\text { santé dans le contexte } \\
\text { de la migration et de } \\
\text { l'asile? }\end{array}$ & $\begin{array}{l}\text { - Traduction/soutien linguistique } \\
\text { - Mesures de socialisation et activités } \\
\text { occupationnelles } \\
\text { - Education et sensibilisation } \\
\text { (aux soins, au fonctionnement } \\
\text { du système de santé) } \\
\text { - Empowerment } \\
\text { - Médecin traitant fixe pour continuité } \\
\text { des soins } \\
\text { - Militantisme positif } \\
\text { - Travail en réseau }\end{array}$ & $\begin{array}{l}\text { - Synergie et interdisciplinarité } \\
\text { - Structures spécialisées } \\
\text { - Formation du personnel } \\
\text { (compétences transculturelles) } \\
\text { - Conscientisation des biais et change- } \\
\text { ments des comportements } \\
\text { - Financement (transports et frais } \\
\text { de traductions gratuits) } \\
\text { - Interprétariat communautaire } \\
\text { accessible et de qualité }\end{array}$ & $\begin{array}{l}\text { - Déclarations internationales } \\
\text { (telle que déclaration de Budapest }{ }^{1} \text { ) } \\
\text { - Modification du cadre légal } \\
\text { - Financement de projets innovants } \\
\text { - Collaboration avec la société civile } \\
\text { - Implication de la communauté } \\
\text { - Assurance maladie } \\
\text { - Adaptation du dépistage à l'épidémio- } \\
\text { logie }\end{array}$ \\
\hline
\end{tabular}

${ }^{1}$ Déclaration de Budapest Sur les Droits, la Santé et le Bien-être des Enfants et des Jeunes Déplacés (https://www.issop.org/cmdownloads/budapest-declaration-french-version/). 
langue, le statut socio-économique, l'isolement social, le cadre légal et administratif, la formation du personnel (en particulier des compétences cliniques transculturelles), le financement et la continuité des soins. A ces similitudes s'ajoutent de nombreux enjeux, solutions et barrières spécifiques à une sous-population (le sexisme et les violences sexuelles dont sont victimes les femmes migrantes par exemple), une situation sociale (stigmatisation et méconnaissance des personnes emprisonnées) ou un problème de santé (aspect culturel et de langage dans le contexte des maladies psychiques). Le détail complet de cette synthèse est présenté sous forme de tableau (cf. tableau).

\section{Nécessité de regrouper les acteurs}

La réalisation de tels séminaires permet de réunir les différents acteurs autour de la thématique de l'équité des soins afin d'échanger, partager et synthétiser les principaux enjeux et esquisser des pistes de solutions. Un élément clé - également ressorti du Symposium réside dans la nécessité de la collaboration interprofessionnelle, en particulier dans un domaine comme l'asile et la migration, où le cadre légal ou politique est parfois insatisfaisant pour garantir une prise en charge optimale et durable. Ce genre de conférence semble essentiel pour se former et s'engager auprès de populations cibles aux besoins de santé spécifiques. La tenue d'un symposium est un outil important de réseautage, de formation et de renforcement de l'ensemble des personnes actives et intéressées à améliorer l'accès et la qualité des soins pour toutes et tous. Certaines conclusions de l'événement se retrouvent dans les tra- vaux menés par les chercheurs canadiens de «Equip Health Care» dont le but est de renforcer l'équité au sein du système de santé, en particulier les notions de structures adaptées, de formations spécifiques, de prise en compte des déterminants sociaux de la santé et d'implication du patient [7]. Le prochain Symposium romand sur l'équité des soins aura lieu le 12 juin 2020. Il se concentrera sur l'orientation sexuelle et l'identité de genre.

\section{Remerciements}

Nous tenons à remercier tout particulièrement l'ensemble de l'équipe d'organisation du Symposium, à savoir: Brigitte Pahud, Patricia Sylvestre, Heidi Abelairas, Nezha Gysler, Caroline San Miguel et Benoît Perrault. Merci aussi à Emilie Jendly du service de communication d'Unisanté pour sa collaboration.

Nous remercions également tous les intervenants pour leur participation et la qualité de leurs présentations. Enfin, nous remercions le CHUV pour la mise à disposition des locaux.

\section{Références}

1 World Health Organization [en ligne]. Health systems, Equity [cité le 10 octobre 2019] [Available from: https://www.who.int/ healthsystems/topics/equity/en/].

2 Marmot M, Friel S, Bell R, Houweling TAJ, Taylor S, Hlt CSD. Closing the gap in a generation: health equity through action on the social determinants of health. Lancet. 2008;372(9650):1661-9.

3 Marmot M, Bell R. The Sustainable Development Goals and Health Equity. Epidemiology. 2018;29(1):5-7.

4 Marmot M. The health gap: the challenge of an unequal world. 2015 Dec 12. p. 2442-4.

5 Cookson R, Asaria M, Ali S, Ferguson B, Fleetcroft R, Goddard M, et al. Health Equity Indicators for the English NHS: a longitudinal whole-population study at the small-area level. Health Services and Delivery Research. Southampton (UK) 2016.

6 Patrick Bodenmann YJ, Hans Wolff. Vulnérabilités, équité et santé. Hygiène RéMe, editor. Suisse 2018. 430 p.

7 Browne AJ, Varcoe C, Ford-Gilboe M, Wathen CN, Team ER. EQUIP Healthcare: An overview of a multi-component intervention to en hance equity-oriented care in primary health care settings. Int J Equity Health. 2015;14:152.
Prof. Patrick Bodenmann Centre universitaire de médecine générale et santé publique (Unisanté) Département Vulnérabilités et médecine sociale (DVMS) Rue du Bugnon 44 CH-1011 Lausanne patrick.bodenmann[at] unisante.ch

\section{Das Wichtigste in Kürze}

- Pflegeverzicht aus finanziellen Gründen, häufige Nutzung von Notfällen: Die Anzeichen von Ungleichheiten im schweizerischen Gesundheitssystem werden immer wichtiger. Personen mit Migrationshintergrund sind besonders betroffen.

- Allerdings fehlen noch viele Daten, um ein echtes Verständnis von Gleichgerechtigkeitsfragen in einer Gemeinde oder einem Gesundheitssystem zu ermöglichen.

- Daher hat das Bundesamt für Gesundheit das «Centre universitaire de médecine générale et santé publique (Unisanté)" beauftragt, das erste Symposium über Gesundheitsgerechtigkeit in der Westschweiz mit Fokus auf Asyl zu organisieren. Unisanté verfügt über ein Fachwissen im Bereich der Migration.

- Während der Tagung diskutierten die 250 anwesenden Gesundheitsfachpersonen Fragen, Hindernisse und Lösungen im Zusammenhang mit der Gesundheitsgerechtigkeit im Asylbereich.

\section{L'essentiel en bref}

- Les soins ne sont pas équitablement accessibles à toutes et tous en Suisse. Les personnes issues de la migration représentent un groupe particulièrement vulnérable.

- Langue, contexte socio-culturel, coûts, méconnaissance, méfiance sont autant d'obstacles qui empêchent cette population d'accéder au système de santé.

- Pour y remédier, un Symposium romand sur l'équité des soins a réuni différents acteurs liés à cette problématique en juin dernier. La conférence a été mandatée et financée par l'Office fédéral de la santé publique.

- L'un des acteurs est Unisanté, centre universitaire de médecine générale et santé publique, chargé par I'OFSP de davantage sensibiliser à cette thématique au niveau romand. Via son Département Vulnérabilités et médecine sociale, il détient une expertise dans le domaine de l'équité des soins au niveau national. 